\title{
Macrophage depletion in stellate ganglia alleviates cardiac sympathetic overactivation and ventricular arrhythmogenesis by attenuating neuroinflammation in heart failure
}

\author{
Dongze Zhang ${ }^{1} \cdot$ Wenfeng Hu ${ }^{1} \cdot$ Huiyin Tu ${ }^{1} \cdot$ Bryan T. Hackfort ${ }^{2} \cdot$ Bin Duan ${ }^{3} \cdot$ Wanfen Xiong $^{4} \cdot$ Michael C. Wadman $^{1}$. \\ Yu-Long $\mathrm{Li}^{1,2}$
}

Received: 14 February 2021 / Accepted: 13 April 2021 / Published online: 21 April 2021

(c) The Author(s) 2021

\begin{abstract}
Cardiac sympathetic overactivation is involved in arrhythmogenesis in patients with chronic heart failure (CHF). Inflammatory infiltration in the stellate ganglion (SG) is a critical factor for cardiac sympathoexcitation in patients with ventricular arrhythmias. This study aims to investigate if macrophage depletion in SGs decreases cardiac sympathetic overactivation and ventricular arrhythmogenesis in CHF. Surgical ligation of the coronary artery was used for induction of CHF. Clodronate liposomes were microinjected into bilateral SGs of CHF rats for macrophage depletion. Using cytokine array, immunofluorescence staining, and Western blot analysis, we found that macrophage expansion and expression of TNF $\alpha$ and IL- $1 \beta$ in SGs were markedly increased in CHF rats. Flow cytometry data confirmed that the percentage of macrophages in SGs was higher in $\mathrm{CHF}$ rats than that in sham rats. Clodronate liposomes significantly reduced CHF-elevated proinflammatory cytokine levels and macrophage expansion in SGs. Clodronate liposomes also reduced CHF-increased $\mathrm{N}$-type $\mathrm{Ca}^{2+}$ currents and excitability of cardiac sympathetic postganglionic neurons and inhibited CHF-enhanced cardiac sympathetic nerve activity. ECG data from 24-h, continuous telemetry recording in conscious rats demonstrated that clodronate liposomes not only restored CHF-induced heterogeneity of ventricular electrical activities, but also decreased the incidence and duration of ventricular tachycardia/fibrillation in CHF. Macrophage depletion with clodronate liposomes attenuated CHF-induced cardiac sympathetic overactivation and ventricular arrhythmias through reduction of macrophage expansion and neuroinflammation in SGs.
\end{abstract}

Keywords Macrophage depletion · Clodronate liposomes $\cdot$ Calcium channel $\cdot$ Cardiac sympathetic neuron · Ventricular arrhythmia $\cdot$ Chronic heart failure

Dongze Zhang, Wenfeng Hu, Huiyin Tu contributed equally to the study.

$\triangle$ Dongze Zhang

dongze.zhang@unmc.edu

Yu-Long Li

yulongli@unmc.edu

1 Department of Emergency Medicine, University of Nebraska Medical Center, Omaha, NE 68198, USA

2 Department of Cellular and Integrative Physiology, University of Nebraska Medical Center, Omaha, NE 68198, USA

3 Mary \& Dick Holland Regenerative Medicine Program, Division of Cardiology, Department of Internal Medicine, University of Nebraska Medical Center, Omaha, NE 68198, USA

4 Department of Surgery, University of Nebraska Medical Center, Omaha, NE 68198, USA

\section{Introduction}

As a common complication in chronic heart failure (CHF), ventricular arrhythmia accounts for nearly $50-60 \%$ of mortality in patients with CHF [9]. Besides structural and electrophysiological remodeling in the ventricle [49], neuronal remodeling in the autonomic nervous system plays an important role in the development and maintenance of ventricular arrhythmias in CHF [36]. It has been demonstrated that excess cardiac sympathoexciation with the subsequent release of neurotransmitters from nerve terminals is a substrate to evoke ventricular arrhythmias in CHF [9, 25]. However, the management of sympathetic overactivation remains challenging in the clinic. This study attempted to explore a novel therapeutic strategy to inhibit cardiac sympathetic overactivation and ventricular arrhythmias. 
Recent studies have revealed an elevation of inflammatory infiltration and macrophage hyperactivation in stellate ganglion (SG) from patients with cardiomyopathy and arrhythmias $[1,40]$. It remains unclear if and how neuroinflammation in the SG contributes to ventricular arrhythmias. Voltage-dependent calcium channels (VDCCs) play an important role in a wide variety of cell functions through mediating calcium influx [20]. Among various types of VDCCs, N-type calcium (Cav2.2) channels, predominantly expressed in the nervous system, are considered to be essential for modulating neurotransmitter release at sympathetic nerve terminals [20,32]. Our recent study has reported that $\mathrm{N}$-type $\mathrm{Ca}^{2+}$ currents and excitability of cardiac sympathetic postganglionic (CSP) neurons located in the SGs are enhanced in CHF rats [58]. In the meantime, cardiac sympathetic overactivation was accompanied with lethal ventricular arrhythmias in the same CHF model [58]. However, the mechanisms underlying CHF-increased $\mathrm{N}$-type $\mathrm{Ca}^{2+}$ currents and excitability of CSP neurons in the SGs are still unclear. Considering that voltage-gated $\mathrm{Ca}^{2+}$ channels could be modulated by proinflammatory cytokines in various tissues and cells [43, 53], it is possible that CHF-enhanced $\mathrm{N}$-type $\mathrm{Ca}^{2+}$ currents in CSP neurons and cardiac sympathoexcitation are attributed to neuroinflammation in the SGs.

Proinflammatory cytokines are produced predominantly by activated macrophages [14]. Depleting macrophages allows researchers to investigate their functions by observing the consequences of cell absence [19]. Clodronate liposomes, phagocytized by macrophages to induce macrophage apoptosis and depletion [42], have been chosen to eliminate macrophages in many studies [21, 52, 55]. By employing clodronate liposomes, some studies emphasized the critical role of brain macrophages in systemic inflammation-triggered central sympathetic excitation in rats with acute myocardial infarction (MI) [55] and hypertension [21]. Thus far, there is no available information about the contribution of neuroinflammation in SGs to cardiac sympathetic overactivation and ventricular arrhythmias in CHF. In the present study, therefore, we microinjected clodronate liposomes into the SGs of CHF rats to test whether local depletion of macrophages in SGs reduces N-type $\mathrm{Ca}^{2+}$ currents and excitability of CSP neurons and subsequently decreases cardiac sympathetic overactivation and ventricular arrhythmogenesis in CHF.

\section{Methods}

The study conformed to guidelines for the Care and Use of Laboratory Animals and was approved by the Institutional Animal Care and Use Committee (IACUC, NO.18-07006-FC) at the University of Nebraska Medical Center. See supplemental materials for detailed description in methods.

\section{Experimental design}

In the present study, a total of 152 male Sprague-Dawley rats (6-7 weeks of age, 180-200 g) were used and they were randomly assigned to sham or CHF group. CHF rats underwent surgical left anterior descending coronary artery (LAD) ligation for induction of MI-related CHF, and sham rats underwent the same surgery without LAD ligation. Implantation of radiotelemetry and labeling of CSP neurons were performed at 11 weeks post-MI. Then, CHF rats were divided into three subgroups for different treatments, including CHF, CHF treated with PBS liposomes, and CHF treated with clodronate liposomes at 12 weeks post-MI. PBS liposomes or clodronate liposomes were microinjected into bilateral SGs. Heart rate variability (HRV), ventricular arrhythmogenesis-related ECG makers, and spontaneous ventricular arrhythmias from 24-h radiotelemetry ECG recording in conscious rats were evaluated in all groups. Terminal experiments including measurement for inducibility of ventricular arrhythmia and cardiac sympathetic nerve activity (CSNA) recording in anesthetized rats, hemodynamic and morphological measurements, echocardiography, Western blot, immunofluorescence staining, cytokine array, flow cytometry, and whole-cell patch-clamp recording in CSP neurons were performed at 13-14 weeks post-MI (Supplemental Fig. 1).

\section{Animal model}

CHF rats were anesthetized with $2 \%$ isoflurane for surgical ligation of the $\mathrm{LAD}$, and sham rats underwent the same surgery without LAD ligation, as described previously $[58,59]$. A Millar pressure transducer was used to determine left ventricle end-diastolic pressure (LVEDP) and systolic pressure (LVSP) in the terminal experiment. CHF was confirmed by multiple morphological and hemodynamic parameters (Table 1).

\section{In vivo microinjection of clodronate liposomes into SGs}

After SGs were identified, clodronate liposomes (Clophosome, $2 \mu \mathrm{l}, 20 \mathrm{mg} / \mathrm{ml}$, CAT: F70101C-AH, FormuMax Scientific Inc, Sunnyvale, CA, USA) or PBS liposomes (CAT: F70101-AH) were microinjected into the bilateral SGs by a glass micropipette. The terminal experiments were performed at 1 week after microinjection.

\section{Implantation of the ECG telemeter and ECG recording in conscious rats}

Implantation of the ECG telemeter was performed as described previously $[3,38,46]$. Under the anesthetized condition, laparotomy was performed at the Linea Alba. 
Table 1 Hemodynamic and morphological characteristics in all groups of rats

\begin{tabular}{lllll}
\hline & $\begin{array}{l}\text { Sham } \\
(n=35)\end{array}$ & $\begin{array}{l}\text { CHF } \\
(n=35)\end{array}$ & $\begin{array}{l}\text { CHF+PBS liposomes } \\
(n=30)\end{array}$ & $\begin{array}{l}\text { CHF+Clo- } \\
\text { dronate } \\
\text { liposomes } \\
(n=30)\end{array}$ \\
\hline Body weight (g) & $411.83 \pm 2.33$ & $410.29 \pm 2.73$ & $409.09 \pm 3.20$ & $413.23 \pm 3.16$ \\
MBP (mmHg) & $101.94 \pm 2.04$ & $101.46 \pm 2.15$ & $102.13 \pm 1.87$ & $103.90 \pm 2.25$ \\
HR (bpm) & $354.46 \pm 4.94$ & $359.60 \pm 5.08$ & $358.73 \pm 5.61$ & $356.97 \pm 6.12$ \\
Infarct size (\% of LV) & 0 & $45.34 \pm 0.63^{*}$ & $45.47 \pm 0.79^{*}$ & $45.33 \pm 0.93^{*}$ \\
LVSP (mmHg) & $122.06 \pm 1.56$ & $98.77 \pm 1.69^{*}$ & $96.30 \pm 1.42^{*}$ & $96.27 \pm 1.67^{*}$ \\
LVEDP (mmHg) & $1.71 \pm 0.11$ & $19.50 \pm 0.47^{*}$ & $19.83 \pm 0.48^{*}$ & $19.68 \pm 0.50^{*}$ \\
\hline
\end{tabular}

Data are means $\pm \mathrm{SE}$

$C H F$ chronic heart failure, $M B P$ mean blood pressure, $H R$ heart rate, $L V$ left ventricle, $L V S P$ left ventricular end-systolic pressure, $L V E D P$ left ventricular end-diastolic pressure

$* p<0.05$ vs. Sham
The ECG transmitter was placed into the abdominal cavity and bipolar electrodes were tunneled subcutaneously. The negative and positive electrodes were secured in underlying tissue near the upper sternal midline and the left side of the xiphoid process, respectively. One week after ECG telemeter implantation, 24-h, continuous ECG recording was performed in conscious rats to quantify the ventricular arrhythmic events including premature ventricular contractions (PVCs) and the ventricular tachycardia/fibrillation (VT/VF). QT and corrected QT (QTc) intervals, as well as T-peak to T-end (Tpe) interval, were calculated from ECG segments in conscious rats.

\section{Measurement of the HRV in conscious rats}

HRV measurement was used to compare sympathetic activation in conscious rats in the current study. For quantification of the HRV, 24-h, continuous ECG signals were acquired in conscious rats. The HRV was analyzed from ECG segments during the $24 \mathrm{~h}$ recording in conscious rats. HRV analysis including low frequency power (LF) from $0.2-0.75 \mathrm{~Hz}$, high frequency power (HF) from $0.75-2.5 \mathrm{~Hz}$, and $\mathrm{LF} / \mathrm{HF}$ ratio was performed in current study [5, 34, 41].

\section{Labeling of SG neurons and whole cell patch-clamp recording for $\mathrm{Ca}^{2+}$ currents and action potentials (APs)}

Considering that SG neurons project to the heart and other target organs, a transported fluorescent dye was used to retrogradely label SG neurons projecting to the myocardium, as described previously $[39,58]$. The isolation of SG neurons for patch-clamp recording was performed at least 1 week after surgery to allow the dye to diffuse to SG neurons. SG neurons were isolated by a two-step enzymatic digestion protocol as described previously $[50,58]$. Only DiI-labeled SG neurons (i.e. CSP neurons) were used to record voltage-gated
$\mathrm{Ca}^{2+}$ currents and APs through the whole cell patch-clamp technique [58]. When the holding potential was $-80 \mathrm{mV}$, a $5-\mathrm{mV}$ step increment between $-60 \mathrm{mV}$ and $60 \mathrm{mV}$ for $500 \mathrm{~ms}$ was used to elicit current-voltage (I-V) relationships. A saturating concentration of $\omega$-conotoxin GVIA $(1 \mu \mathrm{M})[23,50,58]$ was used to block N-type $\mathrm{Ca}^{2+}$ channels in the present study. $\mathrm{N}$-type $\mathrm{Ca}^{2+}$ currents were calculated using an equation: $\mathrm{N}$-type $\mathrm{Ca}^{2+}$ currents $=$ total $\mathrm{Ca}^{2+}$ currents $-\mathrm{Ca}^{2+}$ currents under treatment of $\omega$-conotoxin GVIA $[50,58]$. In current-clamp experiments, a current injection of $100 \mathrm{pA}$ was used to elicit APs, and frequency of APs was measured in a 1-s current clamp.

\section{Measurement of inducibility of ventricular tachyarrhythmia in anesthetized rats}

Surface lead-II ECG was recorded through subcutaneous electrodes under the anesthetized condition. Programmed electrical stimulation (PES) was performed to determine the susceptibility to ventricular tachyarrhythmia in anesthetized rats. A programmed stimulation protocol combined by single (S2), double (S3), or triple extra-stimulus (S4) after a train of eight stimuli $(8 \times \mathrm{S} 1)$ was designed to induce ventricular tachyarrhythmia as described previously $[18,24,46]$. A quotient of ventricular arrhythmia score was used to quantify inducibility of ventricular tachyarrhythmia as described previously [24, 35].

\section{Recording of CSNA in anesthetized rats}

CSNA was recorded in anesthetized rats as described previously $[57,58]$. The left thoracotomy was performed in the second intercostal space and then the left cardiac sympathetic nerve was exposed and dissected distal to the left SG. The central cut end of the cardiac sympathetic nerve was placed on a bipolar platinum electrode for CSNA recording. 


\section{Measurement of hemodynamic and morphological parameters}

Under the anesthetized condition, the right femoral vein and left femoral artery were cannulated for the administration of drugs and monitoring blood pressure and heart rate, respectively. A Millar pressure transducer was inserted into the left ventricle for measurement of LVSP and LVEDP. After in vivo experiments were performed, the rat heart was isolated for measurement of infarct size through a colorimetric technique coupled to a computerized planimetric analysis.

\section{Immunofluorescence staining and Western blot analysis}

For immunofluorescence staining, isolated SGs were fixed and cut into $10 \mu \mathrm{m}$-thick sections at $-20{ }^{\circ} \mathrm{C}$ and then successively incubated with $10 \%$ donkey serum, primary antibodies, and appropriate secondary antibodies for observing cytokine imaging under a laser scanning confocal microscope. For Western blot analysis, protein level was measured using primary antibodies against a target or housekeeping protein and appropriate secondary antibodies. Target protein was normalized by housekeeping protein. Immunofluorescence staining and Western blot analysis were used to determine protein expression of ionized calcium-binding adaptor molecule 1 (Iba1), tumor necrosis factor alpha (TNF $\alpha$ ), and interleukin-1 beta (IL-1 $\beta$ ) in SGs.

\section{Cytokine protein array}

A Rat Cytokine Antibody Array C1 kit was used to screen the levels of cytokine in SGs according to the User Manual. Briefly, the SG sample was added on an array membrane and successively incubated with biotin-conjugated antibody and infrared fluorescent dye-conjugated streptavidin. Finally, the washed membrane was scanned by the infrared image system.

\section{Flow cytometry analyses for macrophage infiltration}

Flow cytometry is a widely used approach for measuring macrophage infiltration or expansion in many studies [28, $29,47]$. Isolated live cells from SGs obtained by enzymatical digestion were incubated with a mix of fluorochromeconjugated antibodies against CD45 and CD11b and then analyzed by a LSRII cell analyzer. Macrophages were identified as $\mathrm{CD} 45^{+} / \mathrm{CD} 11 \mathrm{~b}^{+}$cells $[6,15,45]$.

\section{Echocardiography}

Echocardiography was performed to determine cardiac function [58]. Briefly, B-mode images were acquired in the parasternal long axis under light isoflurane anesthesia. M-mode images were acquired at the level of the left ventricular papillary muscles. Left ventricular end-diastolic diameter (LVDd) and left ventricular end-systolic diameter (LVDs) were measured. Then ejection fraction (EF), fractional shortening (FS), left ventricular end-diastolic volume (LVd Vol), and left ventricular end-systolic volume (LVs Vol) were calculated using standard formulas from the VisualSonics VevoLab software.

\section{Statistical analysis}

All data are presented as means \pm SEM. SigmaPlot 12 was used for data analysis. A student's unpaired $t$-test was used to compare cytokine levels between two groups. A ChiSquare test was used to analyze incidence of ventricular arrhythmias. One-way ANOVA with post-hoc Bonferroni test was used to complete multi-group comparison for other measurements designed in this study. Normal distribution of data was confirmed with Kolmogorov-Smirov test and equal variance with Levene's test. Statistical significance was accepted when $p<0.05$.

\section{Results}

\section{CHF-induced neuroinflammation and macrophage expansion in the SG}

We first used a cytokine protein array to screen 19 inflammatory cytokines in SGs from sham and CHF rats (Supplemental Fig. 2a). The data demonstrated that nine cytokines were detectable, and only proinflammatory cytokines including TNF $\alpha$ and IL- $1 \beta$ were elevated in SGs from CHF rats, compared to sham rats. However, there were no significant differences in anti-inflammatory cytokine (IL-10) and other proinflammatory cytokines including cytokine-induced neutrophil chemoattractants (CICN), granulocyte-macrophage colony-stimulating factor (GM-CSF), IL-1 $\alpha$, lipopolysaccharide-induced CXC chemokine (LIX), and vascular endothelial growth factor (VEGF) between sham and CHF rats (Supplemental Fig. 2b, c). The data from immunofluorescence staining (Fig. 1A) and Western blot (Fig. 1B) further confirmed that TNF $\alpha$ and IL-1 $\beta$ levels in SGs were significantly higher in CHF rats than that in sham rats.

Considering that proinflammatory cytokines are produced predominantly by macrophage activation [14], we then tested if CHF-increased proinflammatory cytokine 
A Immunofluorescence staining for TNFa and IL-1 $\beta$ in the SG

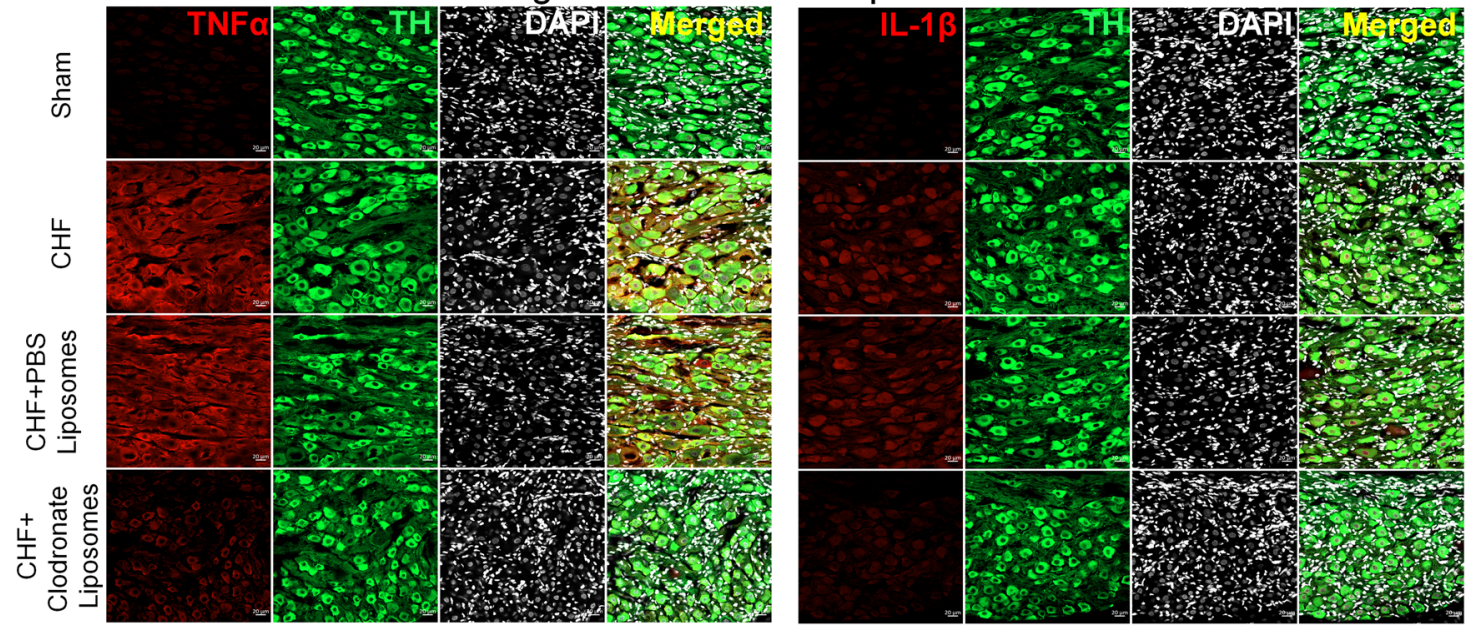

B Western blot data for expression of TNF $\alpha$ and IL-1 $\beta$ in the SG

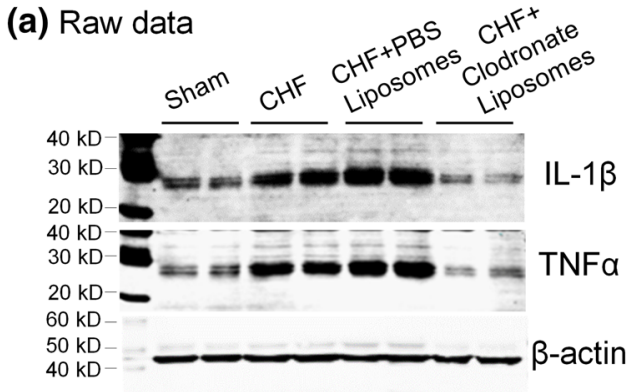

Fig. 1 Macrophage depletion in SGs attenuated the neuroninflammation in CHF. A Representative images of immunofluorescence staining showing increased levels of proinflammatory cytokines including TNF $\alpha$ (left panel) and IL-1 $\beta$ (right panel) in SGs from CHF rat, compared to sham rat. Microinjection of clodronate liposomes reduced CHF-elevated TNF $\alpha$ and IL-1 $\beta$ levels in SGs. DAPI nuclear marker, $\mathrm{TH}$ tyrosine hydroxylase (an adrenergic neuronal marker). B Repre-

levels are attributed to macrophage activation in SGs. Iba1 is a microglial/macrophage-specific calcium-binding protein and it is involved in the membrane phagocytosis in microglial/macrophage $[31,56]$. Considering that it is widely used as a microglial/macrophage marker in mammalian models due to its specific upregulation during the activation of these cells [44, 51], we used the expression of Iba1 protein to determine macrophage activation in SGs. The data about the expression of Iba1 protein showed that macrophages in SGs were markedly activated in CHF rats, compared to age-matched sham rats (Fig. 2A, B). Using Flow cytometry, our data demonstrated that the percentage of macrophages (defined as $\mathrm{CD} 45^{+} / \mathrm{CD} 11 \mathrm{~b}^{+}$cells) in SGs was markedly higher in CHF rats than that in sham rats $(4.60 \pm 0.47 \%$ in $\mathrm{CHF}$ rats vs. $2.07 \pm 0.13 \%$ in sham rats, $p<0.05$, Fig. 2C). sentative images (a) and quantitative data (b) showed expression of TNF $\alpha$ and IL-1 $\beta$ proteins in SGs from all groups, measured by Western blot analysis. Macrophage depletion in SGs markedly decreased expression of TNF $\alpha$ and IL- $1 \beta$ in CHF rats. $n=4$ measurements from 8 rats per group. Statistical significance was determined by oneway ANOVA with post-hoc Bonferroni test. Data are means \pm SEM. ${ }^{*} p<0.05$ vs. sham; ${ }^{\dagger} p<0.05$ vs. CHF

\section{In vivo depletion of macrophages reduced macrophage expansion and neuroinflammation in SGs}

Macrophage expansion and neuroinflammation were measured at 7 days after clodronate liposomes ( $2 \mu \mathrm{l} / \mathrm{side}, 20 \mathrm{mg} /$ $\mathrm{ml}$, an agent for deletion of macrophages) or PBS liposomes (control) were microinjected into bilateral SGs of CHF rats. The immunofluorescence staining (Fig. 2A) and Western blot (Fig. 2B) data demonstrated that clodronate liposomes significantly reduced the CHF-induced macrophage activation. Additionally, data from flow cytometry further confirmed that macrophage expansion in SGs was reduced by clodronate liposomes, as evidenced by the reduction in the percentage of macrophages in CHF plus clodronate liposomes group (Fig. 2C). Clodronate liposomes also attenuated CHF-elevated TNF $\alpha$ and IL-1 $\beta$ levels in SGs (Fig. 1A 
A Immunofluorescence staining for Iba1 in the SG B Western blot data for expression

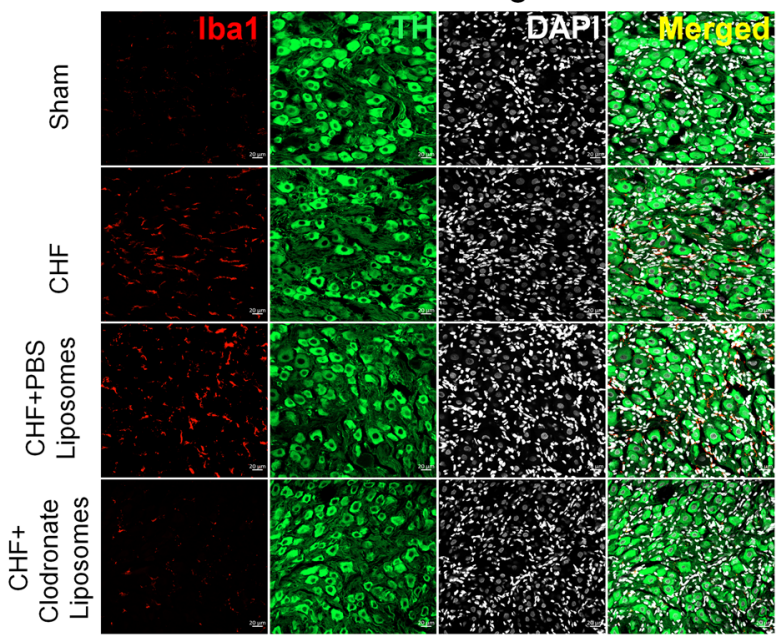

of Iba1 in the SG

(a) Raw data

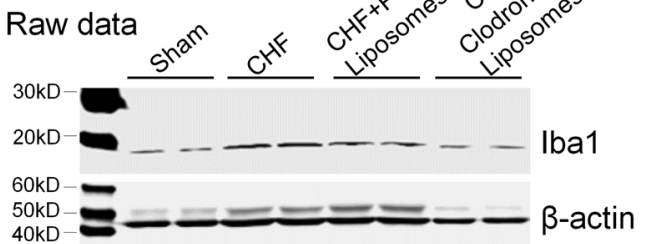

(b) Quantitative data

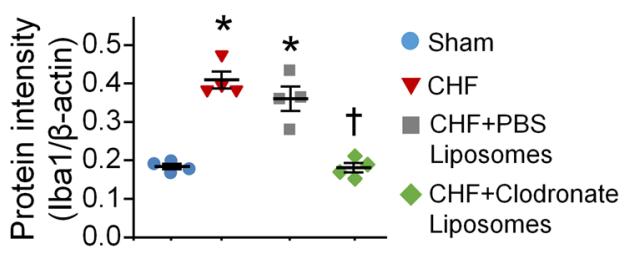

C Flow cytometry data for percentage of macrophages in the SG

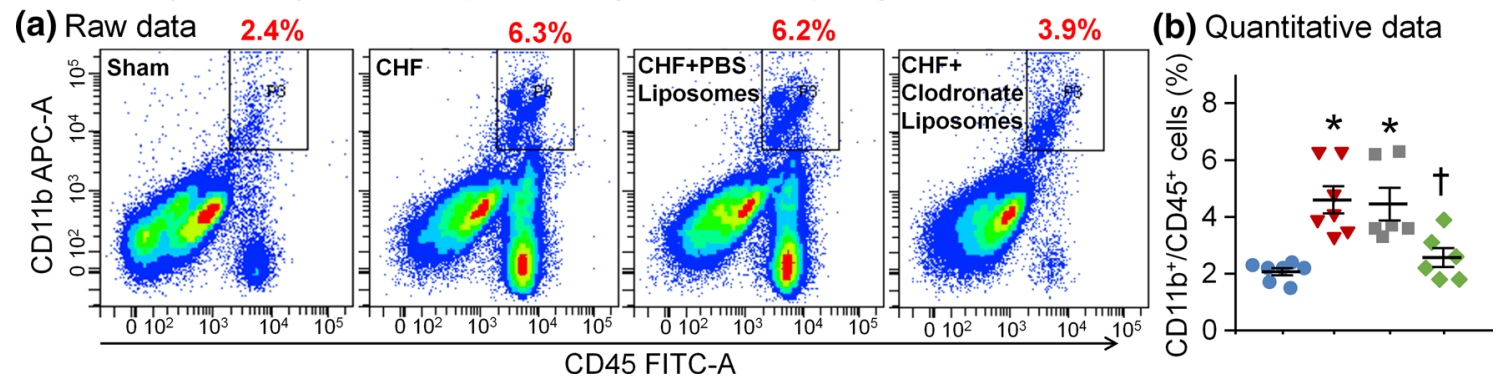

Fig. 2 Microinjection of clodronate liposomes into SGs reduced CHF-elevated macrophage activation in SGs. A Raw images of immunofluorescence staining showing levels of macrophage activation in SGs from all groups. Ibal ionized calcium-binding adaptor molecule 1 protein (a protein marker for macrophage activation), DAPI nuclear marker, TH tyrosine hydroxylase (an adrenergic neuronal marker). B Representative images (a) and quantitative data (b) of Western blot analysis showing CHF-increased expression of Iba1 protein was reduced by clodronate liposomes. $n=4$ measurements

and B). Treatment with PBS liposomes had no effect on CHF-elevated macrophage activation and expansion (Fig. 2), as well as proinflammatory cytokine levels in SGs (Fig. 1).

\section{Macrophage depletion in SGs reduced CHF-increased $\mathrm{N}$-type $\mathrm{Ca}^{2+}$ currents and excitability of CSP neurons}

Using the whole-cell patch-clamp technique, we recorded $\mathrm{N}$-type $\mathrm{Ca}^{2+}$ currents and APs in DiI-labeled SG neurons (i.e. CSP neurons, Fig. 3A). N-type $\mathrm{Ca}^{2+}$ currents were obtained by subtracting $\mathrm{Ca}^{2+}$ currents under treatment of $\omega$-conotoxin GVIA (a specific $\mathrm{N}$-type $\mathrm{Ca}^{2+}$ channel blocker) from total $\mathrm{Ca}^{2+}$ currents (Fig. 3Ba). Compared to sham rats, total $\mathrm{Ca}^{2+}$ currents (Supplemental Fig. 3a), N-type $\mathrm{Ca}^{2+}$ currents (Fig. 3B), and frequency of APs (a parameter of the neuron excitability, Fig. 3c) in CSP neurons were markedly from 8 rats per group. C Representative images (a) and quantitative data (b) for flow cytometry analysis showing macrophage expansion in SGs from all groups. CHF-increased percentage of macrophages (defined as $\mathrm{CD} 45^{+} / \mathrm{CD} 11 \mathrm{~b}^{+}$cells) in SGs was reduced after local treatment with clodronate liposomes. $n=6-7$ rats per group. Statistical significance was determined by one-way ANOVA with post-hoc Bonferroni test. Data are means \pm SEM. $* p<0.05$ vs. sham; ${ }^{\dagger} p<0.05$ vs. CHF

increased in CHF rats, which is consistent with the results from our previous study [58]. Treatment with clodronate liposomes, instead of PBS liposomes, in SGs normalized CHF-increased total $\mathrm{Ca}^{2+}$ currents (Supplemental Fig. 3a), $\mathrm{N}$-type $\mathrm{Ca}^{2+}$ currents (Fig. 3Bc), and frequency of APs (Fig. 3C) towards the level seen in sham rats. Additionally, there were no significant differences in other types (including L-type, $\mathrm{P} / \mathrm{Q}$-type, and R-type) of $\mathrm{Ca}^{2+}$ currents, cell membrane capacitance, input resistance, and resting membrane potential among groups (Supplemental Fig. 3b-e).

\section{Macrophage depletion in SGs attenuated cardiac sympathetic overactivation in both conscious and anesthetized CHF rats}

To assess the effects of macrophage depletion on cardiac sympathetic excitation in CHF rats, we examined 
Fig. 3 Macrophage depletion in SGs reduced CHF-increased $\mathrm{N}$-type $\mathrm{Ca}^{2+}$ currents and excitability of CSP neurons. A Bright field monochrome image of SG neurons (a) and DiI (red color)-labeled SG neurons (i.e. CSP neurons, $\mathbf{b}$ ). B Original recording of $\mathrm{Ca}^{2+}$ currents (a), current-voltage (I-V) curve of $\mathrm{N}$-type $\mathrm{Ca}^{2+}$ currents (b), and quantitative data of $\mathrm{N}$-type $\mathrm{Ca}^{2+}$ currents elicited by $500-\mathrm{ms}$ test pulse at $0 \mathrm{mV}$ from holding potential of - $80 \mathrm{mV}$ (c) in CSP neurons from all groups. C Original recording of action potentials (APs, a) and quantitative data for frequency of APs (b) in CSP neurons from all groups. In vivo microinjection of clodronate liposomes into SGs attenuated CHF-increased $\mathrm{N}$-type $\mathrm{Ca}^{2+}$ currents and excitability of CSP neurons. $n=8$ neurons from 6 rats per group. Statistical significance was determined by one-way ANOVA with post-hoc Bonferroni test. Data are means \pm SEM. $* p<0.05$ vs. sham; ${ }^{\dagger} p<0.05$ vs. CHF
A Raw image for

Dil-labeled SG neuron

(a) Bright field

(b) Dil image
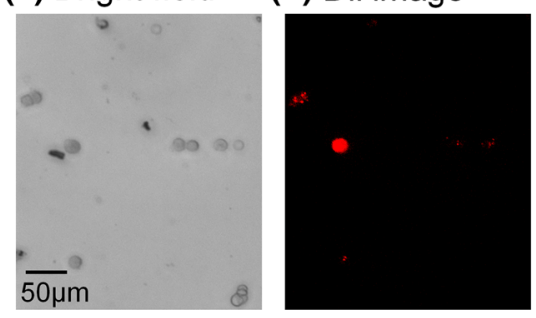

(b) I-V curve

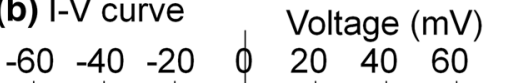

B $\mathrm{Ca}^{2+}$ currents

(a) Raw data

Sham

Total $\mathrm{Ca}^{2+}$ currents

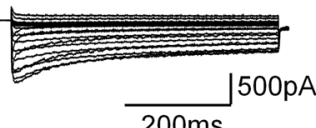

$\mathrm{CHF}$

Total $\mathrm{Ca}^{2+}$ currents

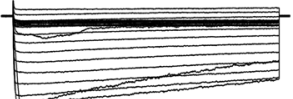

$1 \mu \mathrm{M} \omega$-conotoxin

$1 \mu \mathrm{M} \omega$-conotoxin GVIA

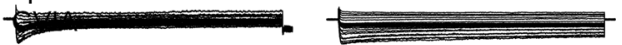

$\mathrm{N}$-type $\mathrm{Ca}^{2+}$ currents $\mathrm{N}$-type $\mathrm{Ca}^{2+}$ currents

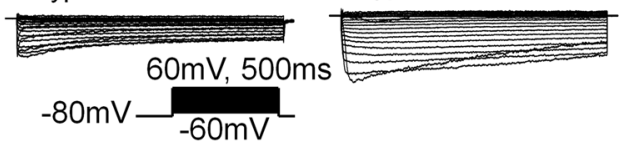

(c) Quantitative data
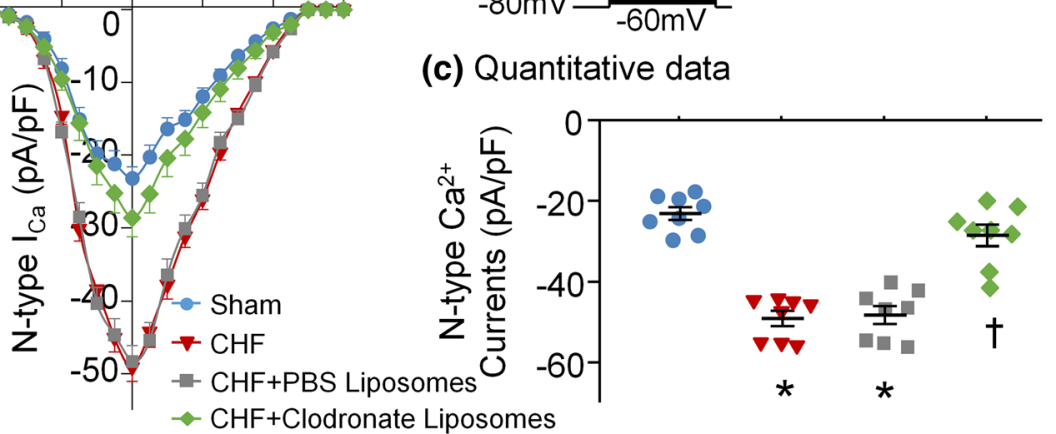

c Action potential (AP)

(a) Raw data

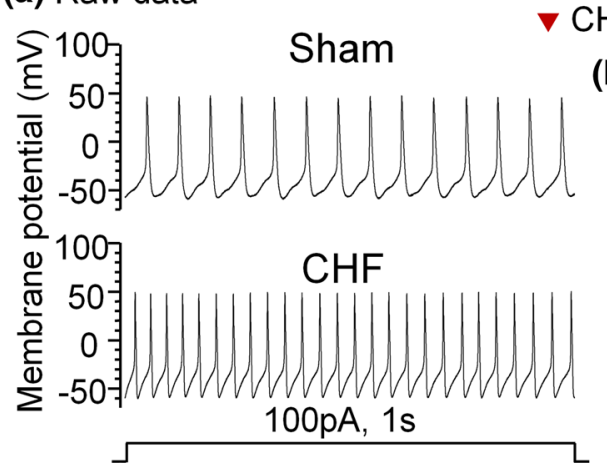

\section{Sham $\quad$ CHF+PBS Liposomes CHF CHF+Clodronate Liposomes}

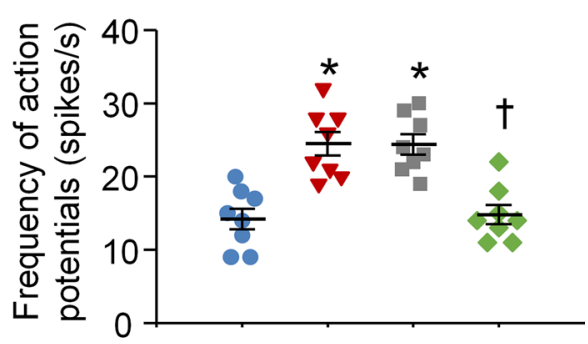

the CSNA and LF/HF ratio of HRV spectral analysis, two indexes of cardiac sympathetic activation. In vivo microinjection of clodronate liposomes into SGs significantly decreased the CSNA in anesthetized CHF rats (Fig. 4A) and the LF and LF/HF ratio in conscious $\mathrm{CHF}$ rats (Fig. 4Bc and $\mathrm{Be}$ ). However, clodronate liposomes did not affect HF (an index of cardiac parasympathetic oscillation) in conscious CHF rats (Fig. 4Bd). Additionally, PBS liposomes had no effects on CSNA, LF, HF, and LF/HF ratio in CHF rats (Fig. $4 \mathrm{~A}$ and $\mathrm{B}$ ).

\section{Improved heterogeneity of ventricular electrical activity after macrophage depletion in SGs in CHF}

Growing evidence demonstrates that heterogeneity of ventricular electrical activities including QT and QTc intervals, QT and QTc dispersions, and Tpe interval plays an important role in ventricular arrhythmogenesis [2]. Like our previous study [58], the data from 24-h ECG recording in conscious rats showed that CHF significantly prolonged QT and QTc intervals, increased QT and QTc dispersions, and lengthened 
Fig. 4 Macrophage depletion in SGs attenuated CHF-induced cardiac sympathetic overactivation in both conscious and anesthetized CHF rats. A Representative tracings (a) and quantitative data (b) for CSNA recorded in all groups of anesthetized rats. Microinjection of clodronate liposomes into SGs markedly reduced CSNA in CHF rats. B Representative $(\mathbf{a}-\mathbf{b})$ and quantitative (c-e) data of HRV analyzed from 24-h ECG recording in conscious rats. Spectral power was quantified for $\mathrm{LF}$ from $0.2-0.75 \mathrm{~Hz}$ and $\mathrm{HF}$ from 0.75 $2.5 \mathrm{~Hz}$. Clodronate liposomes attenuated CHF-enhanced LF and $\mathrm{LF} / \mathrm{HF}$ ratio. $\mathrm{LF}$ and $\mathrm{LF} /$ $\mathrm{HF}$ ratio (indexes of cardiac sympathetic activation) were markedly reduced by clodronate liposomes treated in SGs in CHF. $n=6$ rats per group. Statistical significance was determined by one-way ANOVA with post-hoc Bonferroni test. Data are means \pm SEM. ${ }^{*} p<0.05$ vs. sham; ${ }^{\dagger} p<0.05$ vs. CHF
A Cardiac sympathetic nerve activity (CSNA)
(a) Raw data
(b) Quantitative data
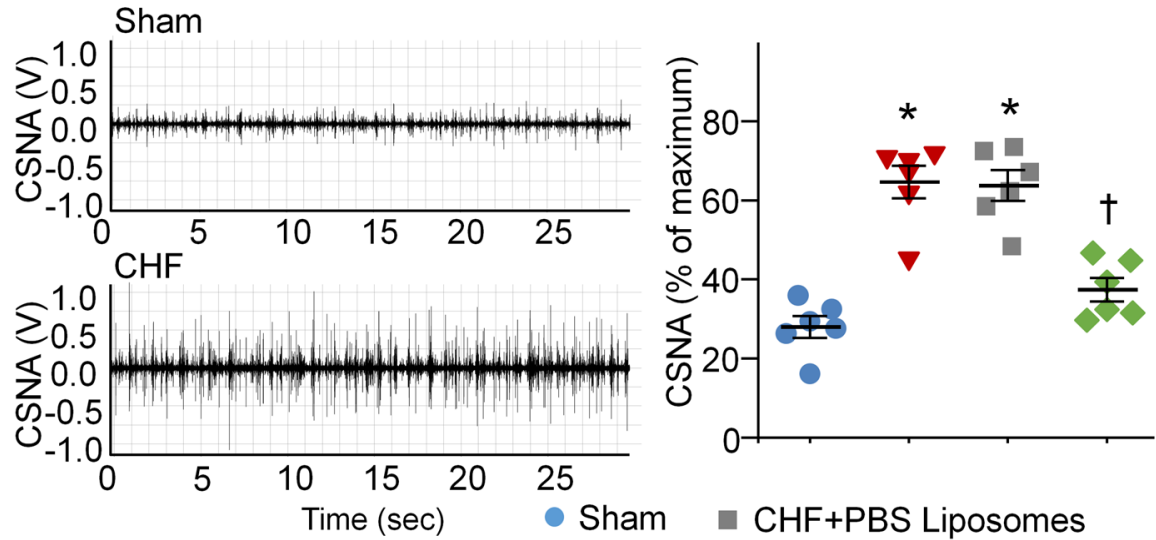

B Heart rate variability (HRV) $\quad \nabla \mathrm{CHF} \quad \mathrm{CHF}+$ Clodronate Liposomes
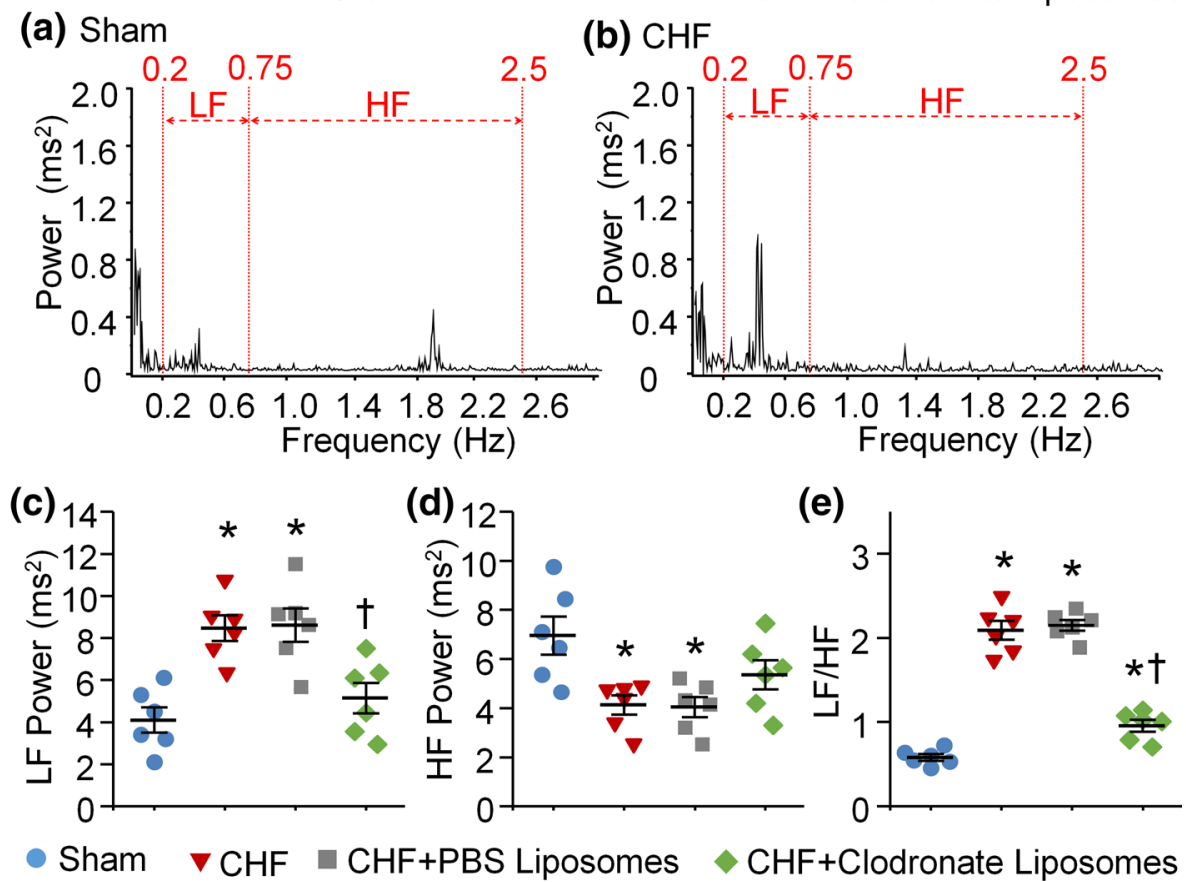

Tpe interval (Supplemental Fig. 4). Treatment with clodronate liposomes in SGs markedly shortened CHF-caused prolongation of QT and QTc intervals, increases in QT and QTc dispersions, and elongation of Tpe interval (Supplemental Fig. 4). However, treatment with PBS liposomes in SGs failed to restore CHF-increased heterogeneity of ventricular electrical activity (Supplemental Fig. 4).

\section{In vivo macrophage depletion in SGs attenuated ventricular arrhythmias in both conscious and anesthetized CHF rats}

In conscious rats, spontaneous ventricular arrhythmias were monitored and analyzed by $24-\mathrm{h}$ radiotelemetry ECG recording (Fig. 5A). In sham rats, PVCs and VT/VF were not detected. In CHF rats, $100 \%$ (6/6) and $83.3 \%(5 / 6)$ of the animals had PVCs and VT/VF, respectively. The number of PVCs and cumulative duration of VT/VF were significantly increased in CHF rats, compared to sham rats (Fig. 5Ac and $\mathrm{Ae}$ ). Microinjection of clodronate liposomes in SGs markedly reduced the incidence and cumulative duration of VT/ $\mathrm{VF}$ and the number of PVCs, but it had no effect on the incidence of PVCs (Fig. 5A).

Besides spontaneous ventricular arrhythmias in conscious rats, PES-triggered inducibility of ventricular arrhythmias (including the incidence of VT/VF and inducibility quotient) was tested in anesthetized rats to further clarify the anti-arrhythmic effect of macrophage 
Fig. 5 Macrophage depletion in SGs alleviated ventricular arrhythmias in both conscious and anesthetized CHF rats. A Raw ECG recordings for PVCs and VT/VF in conscious sham and $\mathrm{CHF}$ rats (a), and mean data for incidence of PVCs (b), the number of PVCs (c), incidence of VT/VF (d), and cumulative duration of VT/VF (e) in all groups of conscious rats. Treatment with clodronate liposomes in SGs significantly reduced the number of PVCs, incidence of $\mathrm{VT} / \mathrm{VF}$, and cumulative duration of VT/VF in CHF rats. B Raw data for PES-evoked VT/ $\mathrm{VF}$ in anesthetized sham and CHF rats (a), and mean data for incidence (b) and inducibility quotient (c) of PES-evoked VT/ VF in all groups of anesthetized rats. Macrophage depletion with clodronate liposomes in SGs markedly decreased incidence and inducibility quotient of PES-evoked VT/VF in anesthetized CHF rats. $n=6$ rats per group. Statistical significance was determined by a Chi-Square test for data presented in panel $\mathrm{Ab}, \mathrm{Ad}$, and $\mathrm{Bb}$. Statistical significance was determined by one-way ANOVA with post-hoc Bonferroni test for data presented in panel Ac, Ae, and $\mathrm{Bc}$. Data are means $\pm \mathrm{SEM}$. $* P<0.05$ vs. sham; ${ }^{\dagger} p<0.05$ vs. $\mathrm{CHF}$
A Spontaneous ventricular arrhythmia in conscious rats

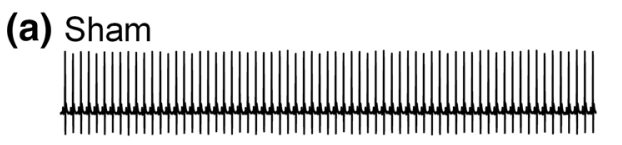

(b) $\square$ No spontaneous PVC - Spontaneous PVC

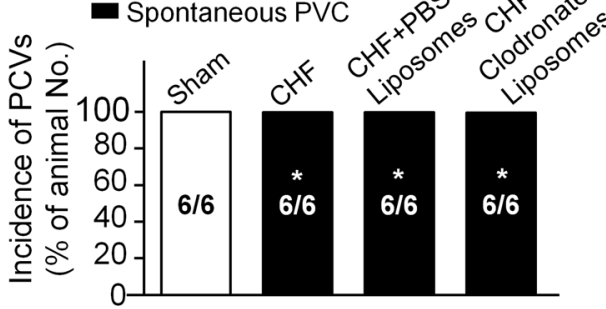

(d) $\square$ No spontaneous VT/NF

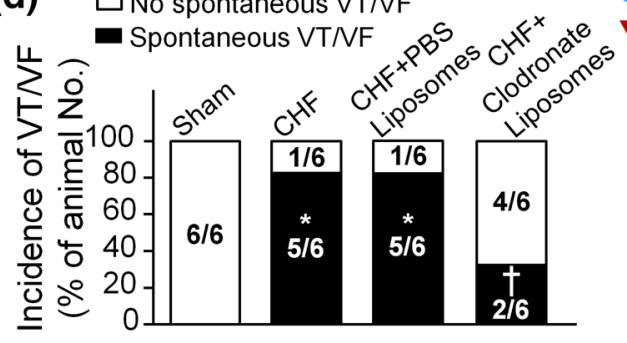

B Inducibility of ventricular tachyarrhythmia in anesthetized rats

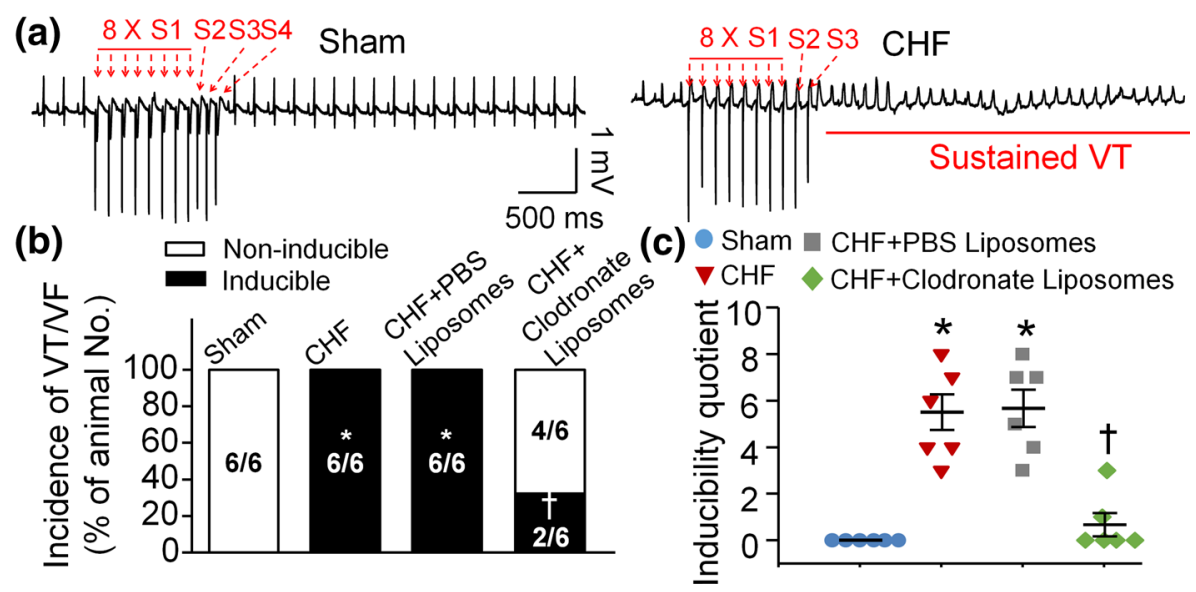

depletion in CHF (Fig. 5B). In sham rats, PES did not trigger the occurrence of VT/VF, and the inducibility quotient was zero. In CHF rats, PES induced VT/VF with a high incidence (100\%) and inducibility quotient $(5.33 \pm 0.80)$, compared to sham rats. Treatment with clodronate liposomes in SGs markedly decreased the incidence of VT/VF (33.3\%) and inducibility quotient $(0.67 \pm 0.49)$ in CHF rats (Fig. 5B). However, treatment with PBS liposomes in SGs had no effect on ventricular arrhythmias in both conscious (Fig. 5A) and anesthetized CHF rats (Fig. 5B).

\section{Macrophage depletion in SGs failed to improve the performance of the failing heart}

The data from echocardiography demonstrated that cardiac performance was markedly reduced in CHF rats, compared to sham rats (Fig. 6). In vivo treatment with clodronate liposomes in SGs failed to improve CHF-impaired cardiac contractile function, including EF, FS, LVDd, LVDs, LVd Vol, and LVs Vol (Fig. 6B). Additionally, hemodynamic and morphological data demonstrated that $\mathrm{CHF}$ rats presented a huge infarct size in the left ventricle and left ventricular 
Fig. 6 Macrophage depletion in SGs failed to improve cardiac performance in $\mathrm{CHF}$. A Representative B-model (upper) and M-mode (bottom) echocardiographic images in left ventricles from Sham, CHF, $\mathrm{CHF}+\mathrm{PBS}$ Liposomes, and $\mathrm{CHF}+$ Clodronate Liposomes rat. B Quantitative data $\mathrm{EF}$ (a), FS (b), LVDd (c), LVDs (d), LVd Vol (e), and LVs Vol (f) calculated from M-mode images of parasternal long axis view in all groups. $n=6$ rats per group. Statistical significance was determined by one-way ANOVA with post-hoc Bonferroni test. Data are means $\pm \mathrm{SEM} ; * p<0.05$ vs. sham
A Representative images of M-Mode Echocardiogram

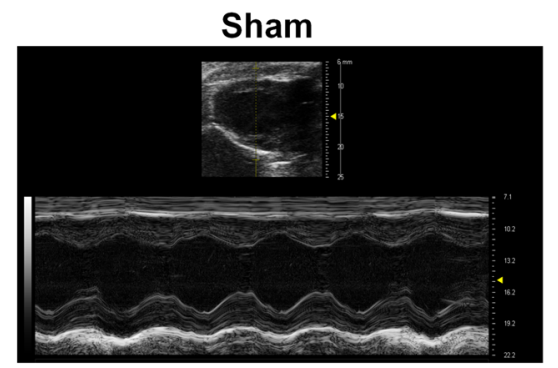

CHF+PBS Liposomes
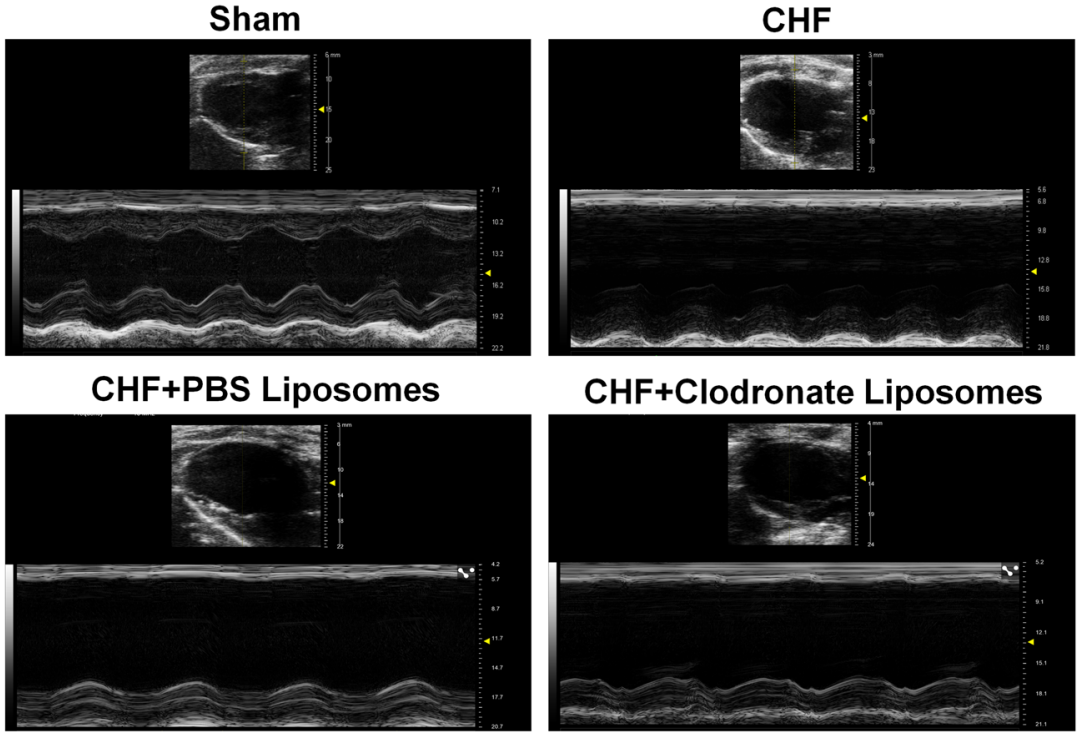

CHF+Clodronate Liposomes

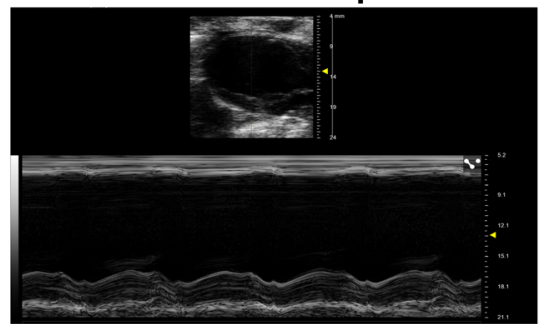

B Quantitative data for cardiac function obtained from Echocardiogram

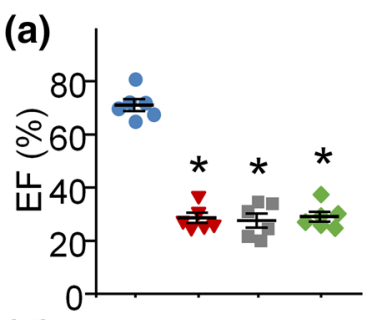

(d)

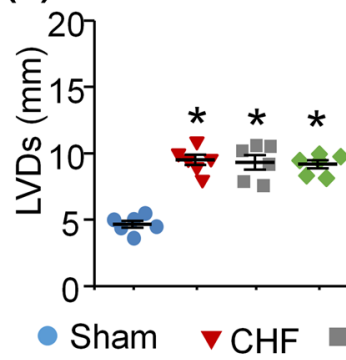

(b)

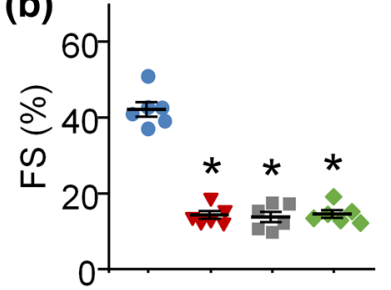

(e)

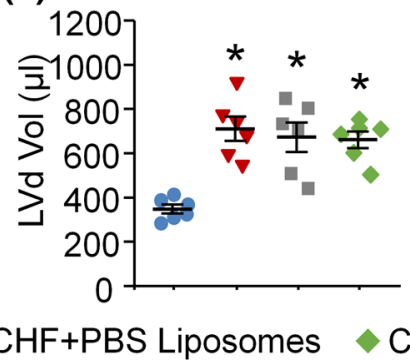

(c) 20

(f)
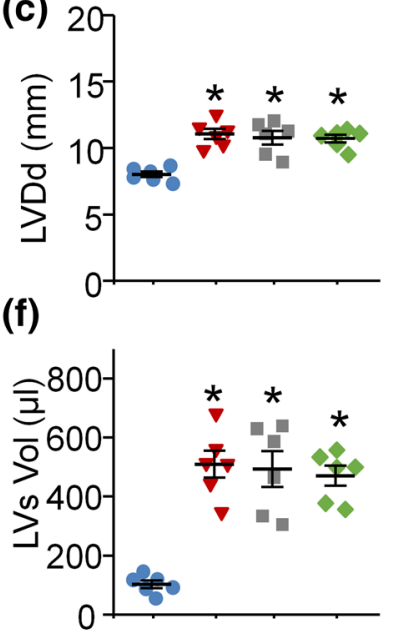

\section{$\mathrm{CHF}+$ Clodronate Liposomes}

contractile dysfunction (Table 1). Treatment with clodronate liposomes in SGs had no effect on hemodynamic and morphological parameters in CHF rats (Table 1). There were no significant differences in body weight, mean blood pressure (MBP), and heart rate (HR) among groups (Table 1).

\section{Discussion}

\section{Major findings}

For the first time, this study provided direct evidence showing the contribution of macrophage activation and its consequence of neuroinflammation in SGs to cardiac sympathetic overactivation and ventricular arrhythmogenesis in the CHF state. As an agent for macrophage deletion, in vivo microinjection of clodronate liposomes into SGs significantly decreased the levels of TNF $\alpha$ and IL- $1 \beta$ in SGs from CHF rats. CHF-increased $\mathrm{N}$-type $\mathrm{Ca}^{2+}$ currents and excitability of CSP neurons were also attenuated by local treatment with clodronate liposomes. Additionally, clodronate liposomes not only blunted cardiac sympathetic overactivation, but also decreased the occurrence of ventricular arrhythmias and heterogeneity of ventricular electrical activities in anesthetized and conscious CHF rats. However, clodronate liposomes failed to improve CHF-induced cardiac contractile dysfunction. Using the strategy of macrophage depletion, our data confirm a direct, positive correlation between 
neuroninflammation in SGs and ventricular arrhythmogenesis in CHF. These data suggest that macrophage depletion or anti-inflammation in SGs could be a potential therapeutic strategy to suppress lethal ventricular arrhythmias and reduce mortality in the CHF state.

\section{Macrophage activation and neuroinflammation in the SG}

MI induces time-dependent changes in the neuroendocrine and expression of various genes [13]. Systemic inflammation after MI indeed serves as the primer for the subsequent occurrence of neuroinflammation in CHF [48]. In the present study, macrophage activation in SGs was confirmed to contribute to the neuroinflammation in the CHF state. From our current study, however, it is unclear why macrophages were over-activated in SGs. Additionally, a combination of CD11b and CD45 labeling is widely used to distinguish microglia from macrophages in many studies $[4,10,12$, 45], which was also employed by our present study to identify macrophages when the cells were double positive with CD45/CD11b. However, it is important to note that CD11b is not only expressed on macrophages, but also expressed on the surface of other leukocytes including monocytes, neutrophils, granulocytes, and natural killer cells [7], and these leukocytes are also $\mathrm{CD} 45^{+}$cells $[26,27]$. Therefore, the above issues including the macrophage overactivation and specific macrophage markers are required to be further identified by future studies.

It has been reported that proinflammatory cytokines such as TNF $\alpha$ and IL-1 are elevated in SGs from patients with a variety of cardiomyopathies and correlated with prognosis and severity of CHF $[11,16]$. However, the direct contribution of neuroinflammation (defined as sustained macrophage activation and production of proinflammatory cytokines) [30] to autonomic dysfunction is not understood. Recently, many studies have greatly recognized the role of neuroinflammation in cardiac sympathetic overactivation and arrhythmogenesis, especially the involvement of inflammatory infiltration in patients with severe ventricular arrhythmias $[1,40]$. Similarly, our current study found that the levels of proinflammatory cytokines including TNF $\alpha$ and IL- $1 \beta$ in SGs were markedly increased in CHF rats. Although the main source of TNF $\alpha$ and IL-1 $\beta$ in SGs was not clarified, several possible sources contributing to CHF-elevated the levels of proinflammatory cytokines in SGs are considered below. First, activated macrophages that originally resided in SGs (converted from microglia) and recruited from circulation (derived from circulating monocytes) are thought to be the key source of proinflammatory cytokines in local tissues [22, 54]. It is supported by our current study that macrophages were markedly activated in SGs from CHF rats (Fig. 2). Second, proinflammatory cytokines recruited from circulation (endocrine fashion) [60] possibly serve as one source for CHF-increased levels of TNF $\alpha$ and IL-1 $\beta$ in SGs. Third, based on the projection of CSP neurons located in SGs to the heart, proinflammatory cytokines retrograded from cardiac myocytes through axons might be another source for CHF-increased levels of proinflammatory cytokines in SGs. One previous study has demonstrated that myocardium-retrograded cytokines could be a key factor to induce CSP neuronal remodeling in SGs [37]. Finally, considering the fact that TNF $\alpha$ itself can promote an M1-like fate in macrophages to trigger the secretion of TNF $\alpha$ and IL-1 $\beta$ from macrophages [8], the interaction among these sources of proinflammatory cytokines could form a vicious circle to elevate local levels of TNF $\alpha$ and IL-1 $\beta$ in SGs from $\mathrm{CHF}$ rats.

\section{Proinflammatory cytokines promote cardiac sympathetic overactivation through affecting $\mathrm{N}$-type $\mathrm{Ca}^{2+}$ channels in CSP neurons in CHF}

Growing evidence has confirmed that VDCCs contribute to cell excitability, intracellular $\mathrm{Ca}^{2+}$ level, and neurotransmitter release in the central and peripheral nervous systems. Thus far, four types (L, N, P/Q, and R) of VDCCs have been reported to be located in CSP neurons. Using human heart atrium, Molderings et al. found that $\mathrm{N}$-type, but not L- and P/Q-type, $\mathrm{Ca}^{2+}$ channels regulate neurotransmitter release from cardiac sympathetic nerve terminals [32]. Our previous study demonstrated that about $60-70 \%$ of whole $\mathrm{Ca}^{2+}$ currents are produced by N-type $\mathrm{Ca}^{2+}$ channels in CPS neurons, and only $\mathrm{N}$-type $\mathrm{Ca}^{2+}$ currents and cell excitability of CSP neurons are increased in CHF rats [50]. More importantly, our recent study used in vivo transfection of $\mathrm{Ca}_{\mathrm{v}} 2.2-\alpha$ shRNA into CSP neurons to normalize CHFincreased $\mathrm{N}$-type $\mathrm{Ca}^{2+}$ currents and cardiac sympathetic activation [58]. Therefore, we believe that CHF-increased $\mathrm{N}$-type $\mathrm{Ca}^{2+}$ currents in CSP neurons could be a key factor for cardiac sympathetic overactivation.

However, the mechanisms responsible for CHF-increased $\mathrm{N}$-type $\mathrm{Ca}^{2+}$ currents in CSP neurons are not understood. The data from RNA sequence analysis demonstrated a significant induction of inflammatory signal in both SGs and dorsal root ganglia following pig chronic MI [13]. Using single-cell patch-clamp recording, Wilkinson et al. found that proinflammatory cytokines such as TNF $\alpha$ and IL- $1 \beta$ activated VDCCs in isolated rat vascular smooth muscle cells [53]. Additionally, neuroinflammation can boost sympathetic activation in both clinical and animal experimental studies $[1,33]$. Our present study demonstrates that CHF induces the elevation of proinflammatory cytokines (TNF $\alpha$ and IL-1 $\beta$ ) mainly derived from activated macrophages in SGs (Figs. 1, 2), which is accompanied by increases in $\mathrm{N}$-type $\mathrm{Ca}^{2+}$ currents of CSP neurons (Fig. 3) and cardiac 
sympathetic overactivation (Fig. 4). In particular, local in vivo microinjection of clodronate liposomes into SGs simultaneously inhibits CHF-induced elevation of TNF $\alpha$ and IL- $1 \beta$, increases in $\mathrm{N}$-type $\mathrm{Ca}^{2+}$ currents, and cardiac sympathetic overactivation. These data provide direct evidence to clarify the involvement of proinflammatory cytokines of CSP neurons in cardiac sympathetic overactivation through affecting the activation of $\mathrm{N}$-type $\mathrm{Ca}^{2+}$ channels in the CHF state. However, our current study cannot answer how proinflammatory cytokines modulate $\mathrm{N}$-type $\mathrm{Ca}^{2+}$ channels, which requires further investigation.

\section{Local depletion of macrophages with clodronate liposomes in SGs is a potential strategy for suppression of ventricular arrhythmias}

Since proinflammatory cytokines are predominantly released from activated macrophages [14], depleting macrophages allows us to test if proinflammatory cytokines contribute to CHF-induced cardiac sympathetic overactivation and ventricular arrhythmias. Systemic injection of clodronate liposomes is perhaps the easiest way to deplete macrophages and has been used for many years [19]. This method relies upon phagocytosis of the clodronate liposomes by macrophages, resulting in the intracellular release of clodronate and subsequent macrophage apoptosis [42]. Given that systemic injection of clodronate liposomes may eliminate macrophages regardless of origin throughout the body [19], an ideal strategy for achieving organ-specific macrophage depletion is local microinjection of clodronate liposomes. This approach has been applied to eliminate perivascular macrophages specifically in the brain [55]. In the present study, therefore, clodronate liposomes were microinjected into SGs for specifically depleting macrophages in SGs. This treatment with clodronate liposomes markedly reduced CHF-elevated macrophage expansion (Fig. 2) and inhibited CHF-increased proinflammatory cytokines including TNF $\alpha$ and IL-1 $\beta$ in SGs (Fig. 1). These data suggest that local depletion of macrophages is an effective therapeutic strategy for anti-inflammation in the SG in CHF.

Using local depletion of macrophages, our current study directly tested whether macrophage activation in SGs aggravates $\mathrm{CHF}$-induced ventricular arrhythmias. Considering the association of systemic inflammation with CHF [48], it is possible that macrophage expansion in other organs such as the brain and heart might be involved in CHF-induced sympathetic overactivation. By macrophage depletion in the central nervous system, Yu et al. reported that proinflammatory cytokines stimulate central sympathetic excitation through activating perivascular macrophages in paraventricular nucleus after acute MI [55]. Although systemic elimination of macrophage has also been reported to suppress cardiac sympathetic hyperinnervation following acute MI [52], the exact pathological contribution of systemic inflammation to cardiac sympathetic excitation and ventricular arrhythmias in the CHF state should be further addressed.

\section{Perspectives and study limitations}

Clinical studies reported that inflammatory infiltration in SGs was significantly increased in patients with severe arrhythmias in the late stage of cardiomyopathy or long QT syndrome $[1,40]$. Our previous study demonstrated that fatal ventricular arrhythmias mainly occur in the early stage (1-3 weeks post- MI) and late stage (12-14 weeks post-MI) of CHF rats [59]. Our present study strongly supports the correlation between neuroinflammation in SGs and severe ventricular arrhythmias in the late stage of some heart diseases demonstrated by clinical studies $[1,40]$. Our study also provides a potential therapeutic strategy that macrophage depletion or anti-inflammation in the late stage of CHF would effectively suppress fatal ventricular arrhythmias through inhibition of macrophage expansion and neuroninflammation in SGs. However, there are some limitations in the current study. First, clodronate liposomes failed to improve the contractile performance of the failing heart in the current study. One reasonable explanation is that clodronate liposomes could not reduce the infarct size and cardiac hypertrophy already formed during the progression of HF when this drug was applied in the late stage of CHF (12 weeks post-MI). Accordingly, we believe that instead of focusing treatment on the developed failing heart, effective pharmacological interventions in proarrhythmic factors such as macrophage expansion and neuroninflammation might be more appropriate in the management of cardiac sympathetic overactivation and ventricular arrhythmias in CHF. Additionally, further studies are needed to clarify whether macrophage depletion or anti-inflammation in the early stage of CHF is beneficial to the failing heart for improvement of cardiac contractile function and suppression of ventricular arrhythmias. Moreover, while treatment with clodronate liposomes in SGs successfully attenuated the CHF-induced cardiac sympathetic overactivation and ventricular arrhythmias, the distinct roles of resident and nonresident macrophages in CHF-induced cardiac sympathetic overactivation and ventricular arrhythmias require further in-depth analyses. Second, although inflammatory infiltration in SGs was measured by immunofluorescence staining, western blot, and flow cytometry in the present study, the histological measurement would provide further morphological support to the increase of macrophages in SGs. Therefore, the histological measurement will be done in future studies as the direct evidence for macrophage infiltration in SGs. Finally, using the dog model of acute myocardial ischemia, Heusch et.al demonstrated that myocardial ischemia increases cardiac sympathetic activity by activating spinal cardiocardiac 
Fig. 7 Contribution of macrophage activation to ventricular arrhythmias in CHF and potential clinical applications. Macrophage activation in SGs aggravates cardiac sympathetic overactivation and ventricular arrhythmias via inducing proinflammatory cytokine-elevated $\mathrm{N}$-type $\mathrm{Ca}^{2+}$ currents and excitability of CSP neurons. Local macrophage depletion with clodronate liposomes in SGs attenuates neuroinflammationactivated $\mathrm{N}$-type $\mathrm{Ca}^{2+}$ channels in CSP neurons, which subsequently reduces $\mathrm{Ca}^{2+}$ influx and CSNA in CHF. Finally, lethal ventricular arrhythmia is suppressed by attenuating cardiac sympathetic overactivationincreased heterogeneity of ventricular electrical activities in CHF. Macrophage depletion and anti-inflammatory treatment in SGs could be a potential therapeutic strategy to suppress cardiac sympathetic overactivation and ventricular arrhythmias in the CHF state

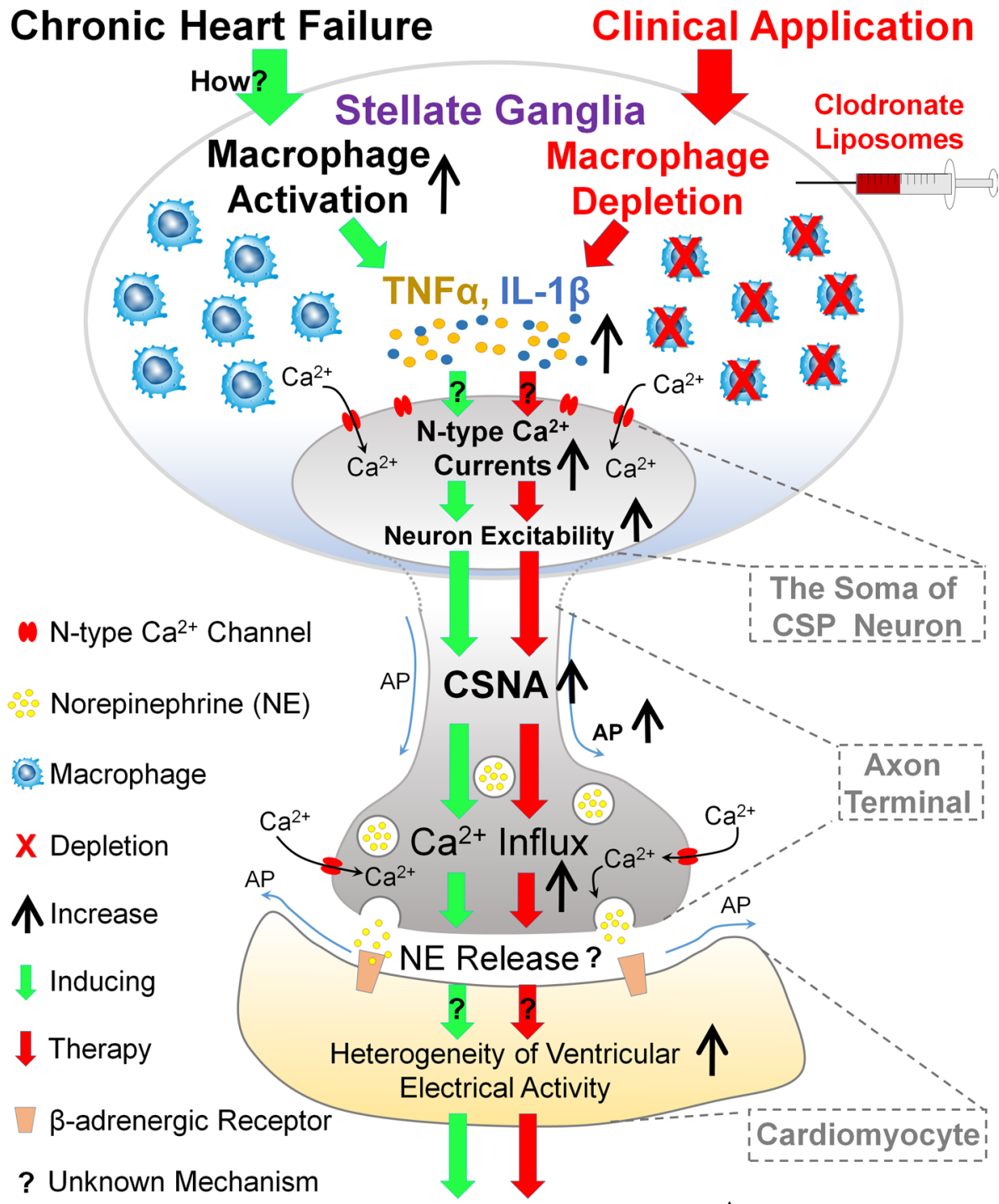

\section{Ventricular Arrhythmia $\uparrow$}

sympathetic reflex [17]. Although our current study demonstrated that CHF-induced cardiac sympathetic overactivation at least partially due to neuroinflammation-activated $\mathrm{N}$-type $\mathrm{Ca}^{2+}$ channels in CSP neurons, this result cannot rule out the involvement of a vicious cycle (positive feedback) between cardiac sympathetic overactivation and severe myocardial ischemia. Further studies are required to explore whether spinal reflex contributes to cardiac sympathetic overactivation during the progression of MI-induced CHF.

\section{Conclusion}

In summary, our present study demonstrates that macrophage depletion with clodronate liposomes in SGs attenuates overactivation of $\mathrm{N}$-type $\mathrm{Ca}^{2+}$ channels and excitability in CSP neurons through reducing the levels of proinflammatory cytokines in SGs, which subsequently decreases cardiac sympathetic overactivation and ventricular arrhythmias in CHF rats (Fig. 7). From these results, we believe that future studies will be designed to access the therapeutic potential of macrophage depletion or antiinflammatory treatment in SGs on-top-of $\beta$-blockers which are widely used in patients with CHF.

Supplementary Information The online version contains supplementary material available at https://doi.org/10.1007/s00395-021-00871-x.

Author contributions DZ and YLL conceived and designed the experiments. DZ, WH, HT, BTH, BD, WX, and YLL performed the experiments. DZ, WH, HT, and YLL analyzed the data. YLL and MCW contributed reagents/materials/analysis tools. DZ and YLL wrote the paper.

Funding This study was supported by the National Institute of Health's National Heart, Lung, and Blood Institute (R01HL-098503, 
R01HL-137832, and R01HL-144146 to Y.L. Li) and University of Nebraska Collaboration Initiative Seed Grant (to B. Duan and Y.L. Li).

Data availability The datasets generated and/or analyzed during the current study are not publicly available due to confidentiality reasons but are available from the corresponding author on reasonable request.

\section{Declarations}

Conflict of interest The authors declare they have no conflicts of interest.

Ethical approval All experimental procedures were approved by the University of Nebraska Medical Center (UNMC) Institutional Animal Care and Use Committee and were carried out in accordance with the National Institutes of Health (NIH Publication No. 85-23, revised 1996).

Open Access This article is licensed under a Creative Commons Attribution 4.0 International License, which permits use, sharing, adaptation, distribution and reproduction in any medium or format, as long as you give appropriate credit to the original author(s) and the source, provide a link to the Creative Commons licence, and indicate if changes were made. The images or other third party material in this article are included in the article's Creative Commons licence, unless indicated otherwise in a credit line to the material. If material is not included in the article's Creative Commons licence and your intended use is not permitted by statutory regulation or exceeds the permitted use, you will need to obtain permission directly from the copyright holder. To view a copy of this licence, visit http://creativecommons.org/licenses/by/4.0/.

\section{References}

1. Ajijola OA, Hoover DB, Simerly TM, Brown TC, Yanagawa J, Biniwale RM, Lee JM, Sadeghi A, Khanlou N, Ardell JL, Shivkumar K (2017) Inflammation, oxidative stress, and glial cell activation characterize stellate ganglia from humans with electrical storm. JCI Insight. https://doi.org/10.1172/jci.insight.94715

2. Antzelevitch C (2007) Heterogeneity and cardiac arrhythmias: an overview. Heart Rhythm 4:964-972. https://doi.org/10.1016/j. hrthm.2007.03.036

3. Baltogiannis GG, Tsalikakis DG, Mitsi AC, Hatzistergos KE, Elaiopoulos D, Fotiadis DI, Kyriakides ZS, Kolettis TM (2005) Endothelin receptor-a blockade decreases ventricular arrhythmias after myocardial infarction in rats. Cardiovasc Res 67:647-654. https://doi.org/10.1016/j.cardiores.2005.04.020

4. Brandenburg S, Blank A, Bungert AD, Vajkoczy P (2021) Distinction of microglia and macrophages in glioblastoma: close relatives, different tasks? Int J Mol Sci 22:194. https://doi.org/10. 3390/ijms22010194

5. Carnevali L, Vacondio F, Rossi S, Macchi E, Spadoni G, Bedini A, Neumann ID, Rivara S, Mor M, Sgoifo A (2015) Cardioprotective effects of fatty acid amide hydrolase inhibitor URB694, in a rodent model of trait anxiety. Sci Rep 5:18218. https://doi.org/10. 1038/srep18218

6. Chen Z, Feng X, Herting CJ, Garcia VA, Nie K, Pong WW, Rasmussen R, Dwivedi B, Seby S, Wolf SA, Gutmann DH, Hambardzumyan D (2017) Cellular and molecular identity of tumor-associated macrophages in glioblastoma. Cancer Res 77:2266-2278. https://doi.org/10.1158/0008-5472.CAN-16-2310
7. Christensen JE, Andreasen SO, Christensen JP, Thomsen AR (2001) CD11b expression as a marker to distinguish between recently activated effector $\mathrm{CD} 8(+) \mathrm{T}$ cells and memory cells. Int Immunol 13:593-600. https://doi.org/10.1093/intimm/13.4.593

8. Clarkson BDS, Kahoud RJ, McCarthy CB, Howe CL (2017) Inflammatory cytokine-induced changes in neural network activity measured by waveform analysis of high-content calcium imaging in murine cortical neurons. Sci Rep 7:9037. https://doi.org/10. 1038/s41598-017-09182-5

9. Cygankiewicz I, Zareba W, Vazquez R, Vallverdu M, GonzalezJuanatey JR, Valdes M, Almendral J, Cinca J, Caminal P, de Luna AB, Subita M, en Insuficiencia Cardiaca I, (2008) Heart rate turbulence predicts all-cause mortality and sudden death in congestive heart failure patients. Heart Rhythm 5:1095-1102. https://doi. org/10.1016/j.hrthm.2008.04.017

10. DePaula-Silva AB, Gorbea C, Doty DJ, Libbey JE, Sanchez JMS, Hanak TJ, Cazalla D, Fujinami RS (2019) Differential transcriptional profiles identify microglial- and macrophagespecific gene markers expressed during virus-induced neuroinflammation. J Neuroinflammation 16:152. https://doi.org/10. 1186/s12974-019-1545-x

11. Dick SA, Epelman S (2016) Chronic heart failure and inflammation: what do we really know? Circ Res 119:159-176. https:// doi.org/10.1161/CIRCRESAHA.116.308030

12. Ford AL, Goodsall AL, Hickey WF, Sedgwick JD (1995) Normal adult ramified microglia separated from other central nervous system macrophages by flow cytometric sorting. Phenotypic differences defined and direct ex vivo antigen presentation to myelin basic protein-reactive CD4+ T cells compared. J Immunol 154:4309-4321

13. Gao C, Howard-Quijano K, Rau C, Takamiya T, Song Y, Shivkumar K, Wang Y, Mahajan A (2017) Inflammatory and apoptotic remodeling in autonomic nervous system following myocardial infarction. PLoS ONE 12:e0177750. https://doi.org/10. 1371/journal.pone.0177750

14. Grumezescu AM (2018) Fullerens, graphenes and nanotubes a pharmaceutical approach. Elsevier; William Andrew, Oxford, pp 601-630

15. Hawkins KE, DeMars KM, Alexander JC, de Leon LG, Pacheco SC, Graves C, Yang C, McCrea AO, Frankowski JC, Garrett TJ, Febo M, Candelario-Jalil E (2017) Targeting resolution of neuroinflammation after ischemic stroke with a lipoxin A4 analog: protective mechanisms and long-term effects on neurological recovery. Brain Behav 7:e00688. https://doi.org/10.1002/brb3. 688

16. Heidenreich $P$ (2017) Inflammation and heart failure: therapeutic or diagnostic opportunity? J Am Coll Cardiol 69:1286-1287. https://doi.org/10.1016/j.jacc.2017.01.013

17. Heusch G, Deussen A, Thamer V (1985) Cardiac sympathetic nerve activity and progressive vasoconstriction distal to coronary stenoses: feed-back aggravation of myocardial ischemia. J Auton Nerv Syst 13:311-326. https://doi.org/10.1016/0165-1838(85) 90020-7

18. Hong T, Yang H, Zhang SS, Cho HC, Kalashnikova M, Sun B, Zhang H, Bhargava A, Grabe M, Olgin J, Gorelik J, Marban E, Jan LY, Shaw RM (2014) Cardiac BIN1 folds T-tubule membrane, controlling ion flux and limiting arrhythmia. Nat Med 20:624632. https://doi.org/10.1038/nm.3543

19. Honold L, Nahrendorf M (2018) Resident and monocyte-derived macrophages in cardiovascular disease. Circ Res 122:113-127. https://doi.org/10.1161/CIRCRESAHA.117.311071

20. Ino M, Yoshinaga T, Wakamori M, Miyamoto N, Takahashi E, Sonoda J, Kagaya T, Oki T, Nagasu T, Nishizawa Y, Tanaka I, Imoto K, Aizawa S, Koch S, Schwartz A, Niidome T, Sawada K, Mori Y (2001) Functional disorders of the sympathetic nervous system in mice lacking the alpha 1B subunit (Cav 2.2) of N-type 
calcium channels. Proc Natl Acad Sci USA 98:5323-5328. https:// doi.org/10.1073/pnas.081089398

21. Iyonaga T, Shinohara K, Mastuura T, Hirooka Y, Tsutsui H (2020) Brain perivascular macrophages contribute to the development of hypertension in stroke-prone spontaneously hypertensive rats via sympathetic activation. Hypertens Res 43:99-110. https://doi.org/ 10.1038/s41440-019-0333-4

22. Jenkins SJ, Ruckerl D, Cook PC, Jones LH, Finkelman FD, van Rooijen N, MacDonald AS, Allen JE (2011) Local macrophage proliferation, rather than recruitment from the blood, is a signature of TH2 inflammation. Science 332:1284-1288. https://doi.org/10. 1126/science. 1204351

23. Jeong SW, Wurster RD (1997) Calcium channel currents in acutely dissociated intracardiac neurons from adult rats. J Neurophysiol 77:1769-1778. https://doi.org/10.1152/jn.1997.77.4.1769

24. Kang CS, Chen CC, Lin CC, Chang NC, Lee TM (2009) Effect of ATP-sensitive potassium channel agonists on sympathetic hyperinnervation in postinfarcted rat hearts. Am J Physiol Heart Circ Physiol 296:H1949-1959. https://doi.org/10.1152/ajpheart. 00903.2008

25. Kaye DM, Lefkovits J, Jennings GL, Bergin P, Broughton A, Esler MD (1995) Adverse consequences of high sympathetic nervous activity in the failing human heart. J Am Coll Cardiol 26:1257-1263. https://doi.org/10.1016/0735-1097(95)00332-0

26. Krzywinska E, Cornillon A, Allende-Vega N, Vo DN, Rene C, Lu ZY, Pasero C, Olive D, Fegueux N, Ceballos P, Hicheri Y, Sobecki M, Rossi JF, Cartron G, Villalba M (2016) CD45 isoform profile identifies natural killer (NK) subsets with differential activity. PLoS ONE 11:e0150434. https://doi.org/10. 1371/journal.pone.0150434

27. Lambert C, Preijers F, Yanikkaya Demirel G, Sack U (2017) Monocytes and macrophages in flow: an ESCCA initiative on advanced analyses of monocyte lineage using flow cytometry. Cytometry B Clin Cytom 92:180-188. https://doi.org/10.1002/ cyto.b. 21280

28. Lindborg JA, Niemi JP, Howarth MA, Liu KW, Moore CZ, Mahajan D, Zigmond RE (2018) Molecular and cellular identification of the immune response in peripheral ganglia following nerve injury. J Neuroinflammation 15:192. https://doi.org/10. 1186/s12974-018-1222-5

29. Liu L, Yin Y, Li F, Malhotra C, Cheng J (2017) Flow cytometry analysis of inflammatory cells isolated from the sciatic nerve and DRG after chronic constriction injury in mice. J Neurosci Methods 284:47-56. https://doi.org/10.1016/j.jneumeth.2017. 04.012

30. Meneses G, Gevorkian G, Florentino A, Bautista MA, Espinosa A, Acero G, Diaz G, Fleury A, Perez Osorio IN, Del Rey A, Fragoso G, Sciutto E, Besedovsky H (2017) Intranasal delivery of dexamethasone efficiently controls LPS-induced murine neuroinflammation. Clin Exp Immunol 190:304-314. https://doi.org/ 10.1111/cei.13018

31. Mikkelsen HB, Huizinga JD, Larsen JO, Kirkeby S (2017) Ionized calcium-binding adaptor molecule 1 positive macrophages and HO-1 up-regulation in intestinal muscularis resident macrophages. Anat Rec (Hoboken) 300:1114-1122. https://doi.org/10.1002/ar. 23517

32. Molderings GJ, Likungu J, Gothert M (2000) N-Type calcium channels control sympathetic neurotransmission in human heart atrium. Circulation 101:403-407. https://doi.org/10.1161/01.cir. 101.4.403

33. Mukerjee S, Lazartigues E (2018) Sympathetic nerve activity and neuro-inflammation: Who is in the driver's seat? Acta Physiol (Oxf). https://doi.org/10.1111/apha.13011

34. Murakami M, Niwa H, Kushikata T, Watanabe H, Hirota K, Ono $\mathrm{K}$, Ohba $\mathrm{T}$ (2014) Inhalation anesthesia is preferable for recording rat cardiac function using an electrocardiogram. Biol Pharm Bull 37:834-839. https://doi.org/10.1248/bpb.b14-00012

35. Nguyen T, El Salibi E, Rouleau JL (1998) Postinfarction survival and inducibility of ventricular arrhythmias in the spontaneously hypertensive rat :effects of ramipril and hydralazine. Circulation 98:2074-2080. https://doi.org/10.1161/01.cir.98.19.2074

36. Ogawa M, Zhou S, Tan AY, Song J, Gholmieh G, Fishbein MC, Luo H, Siegel RJ, Karagueuzian HS, Chen LS, Lin SF, Chen PS (2007) Left stellate ganglion and vagal nerve activity and cardiac arrhythmias in ambulatory dogs with pacing-induced congestive heart failure. J Am Coll Cardiol 50:335-343. https://doi.org/10. 1016/j.jacc.2007.03.045

37. Olivas A, Gardner RT, Wang L, Ripplinger CM, Woodward WR, Habecker BA (2016) Myocardial infarction causes transient cholinergic transdifferentiation of cardiac sympathetic nerves via gp130. J Neurosci 36:479-488. https://doi.org/10.1523/JNEUR OSCI.3556-15.2016

38. Opitz CF, Mitchell GF, Pfeffer MA, Pfeffer JM (1995) Arrhythmias and death after coronary artery occlusion in the rat. Continuous telemetric ECG monitoring in conscious, untethered rats. Circulation 92:253-261. https://doi.org/10.1161/01.cir.92.2.253

39. Pardini BJ, Patel KP, Schmid PG, Lund DD (1987) Location, distribution and projections of intracardiac ganglion cells in the rat. J Auton Nerv Syst 20:91-101. https://doi.org/10.1016/01651838(87)90106-8

40. Rizzo S, Basso C, Troost D, Aronica E, Frigo AC, Driessen AH, Thiene G, Wilde AA, van der Wal AC (2014) T-cell-mediated inflammatory activity in the stellate ganglia of patients with ion-channel disease and severe ventricular arrhythmias. Circ Arrhythm Electrophysiol 7:224-229. https://doi.org/10.1161/ CIRCEP.113.001184

41. Rossi S, Fortunati I, Carnevali L, Baruffi S, Mastorci F, Trombini M, Sgoifo A, Corradi D, Callegari S, Miragoli M, Macchi E (2014) The effect of aging on the specialized conducting system: a telemetry ECG study in rats over a 6 month period. PLoS ONE 9:e112697. https://doi.org/10.1371/journal.pone.0112697

42. Rousselet G (2018) Macrophages: methods and protocols. Springer, New Yourk, pp 259-262

43. Sama DM, Norris CM (2013) Calcium dysregulation and neuroinflammation: discrete and integrated mechanisms for age-related synaptic dysfunction. Ageing Res Rev 12:982-995. https://doi. org/10.1016/j.arr.2013.05.008

44. Sasaki Y, Ohsawa K, Kanazawa H, Kohsaka S, Imai Y (2001) Iba1 is an actin-cross-linking protein in macrophages/microglia. Biochem Biophys Res Commun 286:292-297. https://doi.org/10. 1006/bbrc.2001.5388

45. Savarin C, Dutta R, Bergmann CC (2018) Distinct gene profiles of bone marrow-derived macrophages and microglia during neurotropic coronavirus-induced demyelination. Front Immunol 9:1325. https://doi.org/10.3389/fimmu.2018.01325

46. Shiba Y, Fernandes S, Zhu WZ, Filice D, Muskheli V, Kim J, Palpant NJ, Gantz J, Moyes KW, Reinecke H, Van Biber B, Dardas T, Mignone JL, Izawa A, Hanna R, Viswanathan M, Gold JD, Kotlikoff MI, Sarvazyan N, Kay MW, Murry CE, Laflamme MA (2012) Human ES-cell-derived cardiomyocytes electrically couple and suppress arrhythmias in injured hearts. Nature 489:322-325. https://doi.org/10.1038/nature11317

47. Simeoli R, Montague K, Jones HR, Castaldi L, Chambers D, Kelleher JH, Vacca V, Pitcher T, Grist J, Al-Ahdal H, Wong LF, Perretti M, Lai J, Mouritzen P, Heppenstall P, Malcangio M (2017) Exosomal cargo including microRNA regulates sensory neuron to macrophage communication after nerve trauma. Nat Commun 8:1778. https://doi.org/10.1038/s41467-017-01841-5

48. Thackeray JT, Hupe HC, Wang Y, Bankstahl JP, Berding G, Ross TL, Bauersachs J, Wollert KC, Bengel FM (2018) Myocardial inflammation predicts remodeling and neuroinflammation after 
myocardial infarction. J Am Coll Cardiol 71:263-275. https://doi. org/10.1016/j.jacc.2017.11.024

49. Tomaselli GF, Zipes DP (2004) What causes sudden death in heart failure? Circ Res 95:754-763. https://doi.org/10.1161/01. RES.0000145047.14691.db

50. Tu H, Liu J, Zhang D, Zheng H, Patel KP, Cornish KG, Wang WZ, Muelleman RL, Li YL (2014) Heart failure-induced changes of voltage-gated $\mathrm{Ca} 2+$ channels and cell excitability in rat cardiac postganglionic neurons. Am J Physiol Cell Physiol 306:C132-142. https://doi.org/10.1152/ajpcell.00223.2013

51. Vizioli J, Drago F, Lefebvre C (2016) Neuroprotection and immunity in the medicinal leech hirudo medicinalis: what about microglia? In: Ballarin L, Cammarata M (eds) Lessons in immunity. Academic Press, pp 67-78

52. Wernli G, Hasan W, Bhattacherjee A, van Rooijen N, Smith PG (2009) Macrophage depletion suppresses sympathetic hyperinnervation following myocardial infarction. Basic Res Cardiol 104:681-693. https://doi.org/10.1007/s00395-009-0033-3

53. Wilkinson MF, Earle ML, Triggle CR, Barnes S (1996) Interleukin-1beta, tumor necrosis factor-alpha, and LPS enhance calcium channel current in isolated vascular smooth muscle cells of rat tail artery. FASEB J 10:785-791. https://doi.org/10.1096/fasebj.10.7. 8635696

54. Wynn TA, Vannella KM (2016) Macrophages in tissue repair, regeneration, and fibrosis. Immunity 44:450-462. https://doi.org/ 10.1016/j.immuni.2016.02.015

55. Yu Y, Zhang ZH, Wei SG, Serrats J, Weiss RM, Felder RB (2010) Brain perivascular macrophages and the sympathetic response to inflammation in rats after myocardial infarction. Hypertension 55:652-659. https://doi.org/10.1161/HYPERTENSIONAHA.109. 142836

56. Zelikoff JT, Parmalee NL, Corbett K, Gordon T, Klein CB, Aschner M (2018) Microglia activation and gene expression alteration of neurotrophins in the hippocampus following early-life exposure to e-cigarette aerosols in a murine model. Toxicol Sci 162:276286. https://doi.org/10.1093/toxsci/kfx257

57. Zhang D, Liu J, Tu H, Muelleman RL, Cornish KG, Li YL (2014) In vivo transfection of manganese superoxide dismutase gene or nuclear factor kappaB shRNA in nodose ganglia improves aortic baroreceptor function in heart failure rats. Hypertension 63:88-95. https://doi.org/10.1161/HYPERTENSIONAHA.113.02057

58. Zhang D, Tu H, Wang C, Cao L, Hu W, Hackfort BT, Muelleman RL, Wadman MC, Li YL (2021) Inhibition of N-type calcium channels in cardiac sympathetic neurons attenuates ventricular arrhythmogenesis in heart failure. Cardiovasc Res 117:137-148. https://doi.org/10.1093/cvr/cvaa018

59. Zhang D, Tu H, Wang C, Cao L, Muelleman RL, Wadman MC, Li YL (2017) Correlation of ventricular arrhythmogenesis with neuronal remodeling of cardiac postganglionic parasympathetic neurons in the late stage of heart failure after myocardial infarction. Front Neurosci 11:252. https://doi.org/10.3389/fnins.2017. 00252

60. Zhang JM, An J (2007) Cytokines, inflammation, and pain. Int Anesthesiol Clin 45:27-37. https://doi.org/10.1097/AIA.0b013 e318034194e 